\title{
Temperature dependent behavior of the optical gain and electroabsorption modulation properties of an InAs/GaAs quantum dot epistructure
}

\author{
Emmanuel D. Le Boulbar ${ }^{1}$, Lydia Jarvis ${ }^{1}$,David Hayes ${ }^{1}$, Samuel Shutts ${ }^{1}$, Zhibo Li ${ }^{1}$, Mingchu Tang ${ }^{2}$, \\ Huiyun Liu' ${ }^{2}$ Alireza Samani ${ }^{4}$ Peter M. Smowton ${ }^{1}$, and Nicolás Abadía ${ }^{1}$ \\ ${ }^{1}$ School of Physics and Astronomy, Cardiff University, The Parade, Cardiff CF24 3AA, United Kingdom \\ ${ }^{2}$ Institute for Compound Semiconductors, Cardiff University, The Parade, Cardiff CF24 3AA, United Kingdom \\ ${ }^{3}$ Department of Electronic and Electrical Engineering, University College London, London WC1E 7JE, United \\ Kingdom \\ ${ }^{4}$ Quantum Matter Institute, University of British Columbia, Vancouver, British Columbia V6T 1Z4, Canada
}

\begin{abstract}
In this work, the feasibility of a monolithically integrated laser and electroabsorption modulator based on the same active quantum dot epistructure is studied. The net modal gain and the absorption in the modulator were measured using the segmented contact method from $25{ }^{\circ} \mathrm{C}$ to $125{ }^{\circ} \mathrm{C}$. The maximum of the net modal gain active region of the laser decreases from $10 \mathrm{~cm}^{-1}$ at $25^{\circ} \mathrm{C}$ to $3.9 \mathrm{~cm}^{-1}$ at $125^{\circ} \mathrm{C}$. The non-optimized maximum extinction ratio of the modulator, $4.1 \mathrm{dBmm}^{-1}$, is almost constant until $25{ }^{\circ} \mathrm{C}$. The wavelength at which the net modal gain and the change in absorption are maximum, shift with temperature by $0.04 \mathrm{eV}$.
\end{abstract}

\section{INTRODUCTION}

InAs quantum dot (QD) based devices have been extensively studied in the past decades [1]. Most of the work was done in QD lasers with only a few photodetectors and modulators reported. The majority of those devices were studied in a discrete form. Currently, there is not any in-depth study of the monolothic integration of different QD devices towards a final photonic integrated circuit (PIC). To achieve this integration, the growth and fabrication of monolithically integrated QD devices can be significantly simplified if the same QD epistructure is used across different active devices. Two devices that can be integrated are the laser and the modulator. This work determines the degradation with temperate of this solution when using QDs in the active regions.

Lasers based on QDs offer ultra-low threshold currents, high threshold temperature insensitivity [1], and large tolerance to optical feedback [2]. This tolerance may obviate the need of an isolator at the output of the laser. Another interesting property of QD lasers is that they are more tolerant to temperature and radiation than devices based on quantum wells [3], [4]. Furthermore, the operational wavelength of the laser can be tuned using a Distrubuted Feedback (DFB) or Distrubuted Bragg Reflector (DBR) due to the broad gain spectrum of QDs.

One of the most used effects in electroabsortion modulation is the quantum confined Stark effect (QCSE). The QCSE was studied in InAs quantum dots [5-9] and there are several modulator designs [10-11]. To the best of our knowledge, most of them were not optimized to maximize device performance, E.g. Lin et al. demonstrated an InAs QD electroabsorption modulator with a bandwidth of $3.3 \mathrm{GHz}$ at $1300 \mathrm{~nm}$ in [10]. Another example is [11] where a maximum bit-rate of $2.5 \mathrm{Gbs}^{-1}$ is reached.

There are several lasers and few modulators using QDs in the literature. Their properties where studied for each discrete device, often using different quantum dot structures. In this paper, we address the feasibility of monolithically integrating a QD laser and modulator based on the same active QD epistructure and we study its performance with temperature. For this, we measured the net modal gain and the absorption in the same QD epistructure using the segmented contact method. The net modal gain was measured in forward bias while the change in absorption was measured in reverse bias to exploit the QCSE. The behavior of both devices was studied from $25^{\circ} \mathrm{C}(298 \mathrm{~K})$ to $125^{\circ} \mathrm{C}(398 \mathrm{~K})$ to evaluate its performance for potential use in high temperature environments.

\section{DEVICE FABRICATION AND MEASUREMENT METHOD}

The measurements of the net modal gain of the laser and the absorption in the modulator were performed in the same QD epistructure shown in Fig. 1(a). The InAs/GaAs epistructure was grown by molecular beam epitaxy on n-type GaAs. We employed 5 dot-in-a-well layers (DWELLs), with InAs dots areal density of $5 \times 10^{10} \mathrm{~cm}^{-2}$. The 
grown InAs dots are shown in Fig. 1(b). The intrinsic region of the 5 DWELLs and barriers correspond to a total thickness of $300 \mathrm{~nm}$. The QD active region is placed between two $\mathrm{Al}_{0.4} \mathrm{Ga}_{0.6} \mathrm{As}_{\text {c cladding layers. }}$

To perform the measurement of the net modal gain and absorption, the segmented contact method [12] was employed. Gold multi-section contacts were fabricated with a width of $100 \mu \mathrm{m}$ and a length of $300 \mu \mathrm{m}$. The sections were isolated electrically from each other by etching into the p-doped $\mathrm{GaAs}$ and $\mathrm{Al}_{0.4} \mathrm{Ga}_{0.6} \mathrm{As}$ cladding to a total etch depth of $700 \mathrm{~nm}$. Before performing the measurement, the segmented contact device was mounted on a copper block and wire-bonded to a TO8-can.

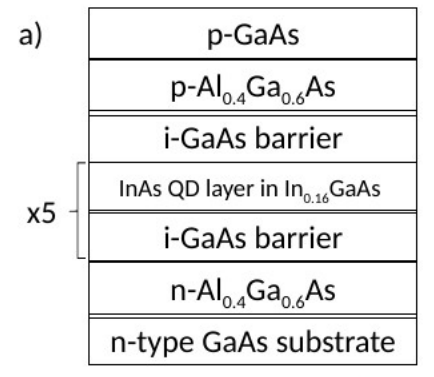

b)

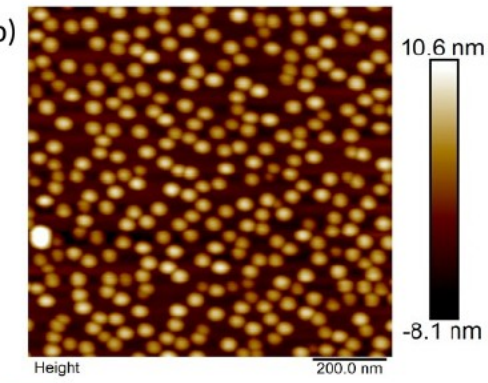

c)

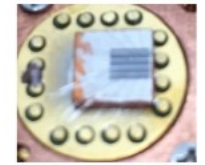

Figure 1: a) schematic representation of the QD epistructure used in this study b), AFM images of QDs before capping of a typical device, $c$ ) image of a the segmented contact device used in this study, mounted on a copper block and wire-bonded to a TO8-can.

\section{GAIN AND ABSORPTION MEASUREMENTS}

In this section, we present the measurements of the monolithically integrated laser and modulator active regions. First, the net modal gain and the absorption of the modulator were measured in the same QD epistructure at room temperature. The measurements are shown in Fig. 2. Then, we studied the behavior of the monolithically integrated active sections from $25^{\circ} \mathrm{C}$ to $125^{\circ} \mathrm{C}$.

The net modal gain for the measurement at room temperature is shown in Fig. 2(a) The forward bias current applied goes from $40 \mathrm{~mA}$ to $180 \mathrm{~mA}$ (133 to $600 \mathrm{~A} \cdot \mathrm{cm}^{-2}$ ) at $25^{\circ} \mathrm{C}$ (298 K). The absorption of the QD active region is shown in Fig. 2 (b. In that figure, the absorption measurement of the same QD epistructure as a function of reverse bias at $25^{\circ} \mathrm{C}(298 \mathrm{~K})$ is shown. It is possible to observe that the absorption at the band edge increases with reverse bias. The QCSE linear redshift goes from $955.8 \mathrm{meV}$ to $946.4 \mathrm{meV}$ and it is in the same order of magnitude found in previous studies [7], [10]. The maximum extinction ratio is $4.1 \mathrm{dBmm}^{-1}$ and it is in agreement with measurements performed in [10] and [11].
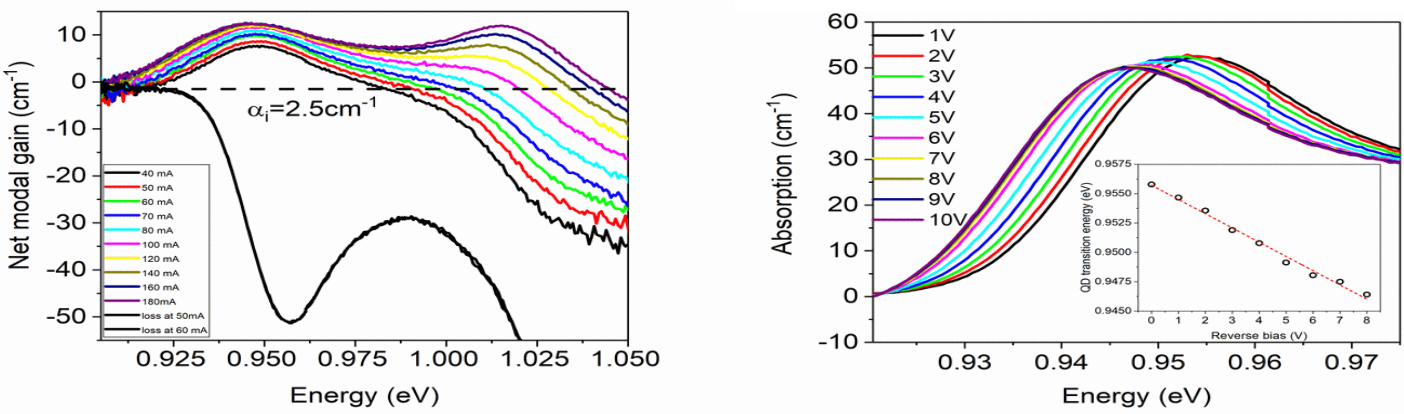

Figure 2: (left) Net modal gain obtained at $25{ }^{\circ} \mathrm{C}$ (298 K) for a range of current and (right) measured absorption for reverse bias from $0 \mathrm{~V}$ to $-10 \mathrm{~V}$. The inset represents the QD transition energy as a function of reverse bias.

In Fig. 2, it is possible to observe that the maximum change in absorption in the modulator and the maximum net modal gain are at slightly different energy. This represented a trade-off between the efficiency of the laser and the efficiency of the modulator. Due to the broad spectrum of the QD active regions, it will be possible to design the DFB or DBR at a slightly different wavelength to select the best trade-off regarding the efficiencies.

Another factor that may influence the total efficiency is the temperature dependence of the band-edge. To study the change in the trade-off between modulation and laser efficiencies we performed the same 
measurements of net modal gain and absorption between $25^{\circ} \mathrm{C}$ to $125^{\circ} \mathrm{C}$. The results are shown in Fig. 3 where the evolution of the net modal gain and the change of absorption $\Delta \alpha_{i}$ are combined. The change in absorption was performed using a voltage sweep $\Delta \mathrm{V}_{\mathrm{pp}}$ of $3 \mathrm{~V}$ at a constant reverse bias of $-3.5 \mathrm{~V}$. To maximize the efficiency, the operational wavelength of the laser will ideally match the wavelength where the highest extinction ratio is obtained in the modulator. The broad gain peak characteristics of QD based structure will be beneficial to match this wavelength. This tuning could be easily achieved by using a DFB or DBR to tune and control the emission wavelength.

Examining the trend of the maximum in the net modal gain, it was found that it decreases with increasing temperature, from $10 \mathrm{~cm}^{-1}$ at $25^{\circ} \mathrm{C}(298 \mathrm{~K})$ to $3.9 \mathrm{~cm}^{-1}$ at $125{ }^{\circ} \mathrm{C}(398 \mathrm{~K})$. The lowest gain of $3.9 \mathrm{~cm}^{-1}$ is sufficient to achieve lasing in a Fabry-Pérot cavity of $3 \mathrm{~mm}$ with $30 \%$ reflective cleaved facets. The measured extinction ratio in the modulator is $4.1 \mathrm{dBmm}^{-1}$. This value is maintained over range 25 to $125{ }^{\circ} \mathrm{C}$. The extinction ratio has a DC reverse bias voltage of $-3.5 \mathrm{~V}$ and a voltage swing $\Delta \mathrm{V}_{\mathrm{pp}}$ of $3 \mathrm{~V}$. The maximum of the net modal gain and the extinction ratio shift with temperature by a similar amount.

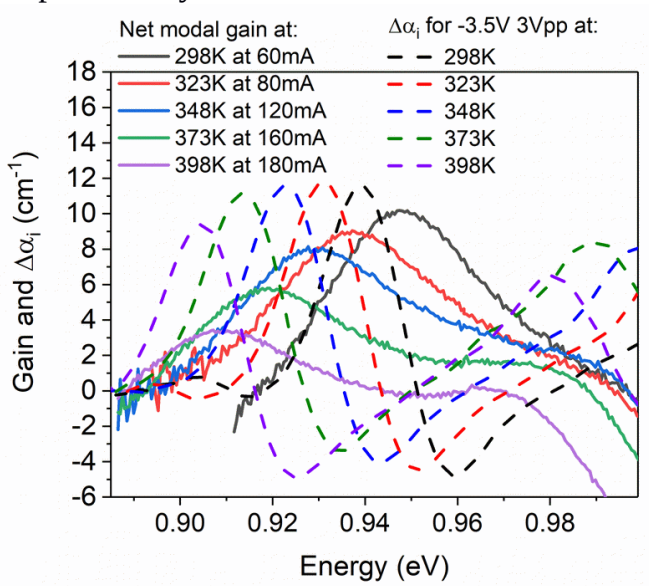

Figure 3: Change in net modal gain and absorption of the QD active section.

\section{CONCLUSION}

We studied the possibility of monolithically integrating a laser and a modulator with the same QD epistructure. Properties of the QD's active region were studied as a function of temperature. The net modal gain was reduced from $10 \mathrm{~cm}^{-1}$ at $25{ }^{\circ} \mathrm{C}$ to $3.9 \mathrm{~cm}^{-1}$ at $125{ }^{\circ} \mathrm{C}$, such values fall within the tolerance sufficient to achieve lasing. Regarding the modulator, it will have a near constant extinction ratio of $4.1 \mathrm{~dB} \cdot \mathrm{mm}^{-1}$ from $25{ }^{\circ} \mathrm{C}$ to $125^{\circ} \mathrm{C}$. The maximum extinction ratio can be further improved when using the QDs in a waveguide. It was found that the wavelength at which the net modal gain is maximum and the wavelength at which the extinction ratio is maximum shifts by a very similar amount. This opens the possibility to use this active region in devices that may operate at high temperatures.

\section{ACKNOWLEDGEMENTS}

We are grateful for support from the Future Compound Semiconductor Manufacturing Hub (CS Hub) funded by EPSRC grant reference EP/P006973/1.

\section{REFERENCES}

[1] M. T. Crowley, N. A. Naderi, H. Su, F. Grillot, and L. F. Lester: 'GaAs-Based quantum dot lasers', in vol. 86. 2012.

[2] H. Huang et al.: 'Analysis of the optical feedback dynamics in InAs/GaAs quantum dot lasers directly grown on silicon', in J. Opt. Soc. Am. B, vol. 35, no. 11, p. 2780, 2018.

[3] F. Guffarth et al.: 'Radiation hardness of InGaAs/GaAs quantum dots', in Appl. Phys. Lett., vol. 82, no. 12, pp. 1941-1943, 2003.

[4] P. G. Piva et al.: 'Enhanced degradation resistance of quantum dot lasers to radiation damage', in Appl. Phys. Lett., vol. 77, no. 5, pp. 624-626, 2000.

[5] C. Y. Ngo et al.: '1.3 $\mu$ m electroabsorption modulator with InAs/InGaAs/GaAs quantum dots, in Appl. Phys. Lett. pp. 15-16, 2009.

[6] C. Y. Ngo et al.: 'Characteristics of $1.3 \mu \mathrm{m}$ InAs/InGaAs/GaAs quantum dot electroabsorption modulator, in Appl. Phys. Lett., vol. 94, no. 14, pp. 1-4, 2009. 
[7] C. Y. Ngo et al.: 'Electroabsorption characteristics of single-mode 1.3- $\mu$ m InAs-InGaAs-GaAs ten-layer quantum-dot waveguide', in IEEE Photonics Technol. Lett., vol. 22, no. 23, pp. 1717-1719, 2010.

[8] D. B. Malins, A. Gomez-Iglesias, E. U. Rafailov, W. Sibbett, and A. Miller: 'Electroabsorption and electrorefraction in an inas quantum-dot waveguide modulator', in IEEE Photonics Technol. Lett., vol. 19, no. 11, pp. 1118-1120, 2007.

[9] I. C. Sandall, J. S. Ng, J. P. R. David, H. Liu, and C. H. Tan: 'Evaluation of InAs quantum dots on Si as optical modulator', in Semicond. Sci. Technol., vol. 28, no. 9, 2013.

[10] C. H. Lin, J. P. Wu, Y. Z. Kuo, Y. J. Chiu, T. E. Tzeng, and T. S. Lay: 'InGaAs self-assembly quantum dot for high-speed $1300 \mathrm{~nm}$ electroabsorption modulator', in J. Cryst. Growth, vol. 323, no. 1, pp. 473-476, 2011.

[11] Y. Chu, M. G. Thompson, R. V Penty, and I. H. White: '1.3 $\mu \mathrm{m}$ Quantum-Dot Electro-Absorption Modulator,' 2007 Conf. Lasers Electro-Optics, pp. 9-10, 2007.

[12] P. Blood, G. M. Lewis, P. M. Smowton, H. Summers, J. Thomson, and J. Lutti: 'Characterization of Semiconductor Laser Gain Media by the Segmented Contact Method', in IEEE J. Sel. Top. Quantum Electron., vol. 9, no. 5, pp. 1275-1282, 2003.

[13] N. Abadía, T. Bernadin, P. Chaisakul, S. Olivier, D. Marris-Morini, R. Espiau de Lamaëstre, J. C. Weeber, and L. Vivien: 'Low-Power consumption Franz-Keldysh effect plasmonic modulator', in Opt. Express, 22 11236-11243, 2014. 